\title{
Geometry of Hydrogen Bonds Formed by Lipid Bilayer Nitroxide Probes: A High Frequency Pulsed ENDOR/EPR Study
}

\author{
Tatyana I. Smirnova ${ }^{1}$, Alex I. Smirnov ${ }^{1}$, Serguei Pachtchenko ${ }^{2}$, and Oleg G. Poluektov ${ }^{2}$ \\ 1 Department of Chemistry, North Carolina State University, 2620 Yarbrough Dr., Raleigh, North Carolina \\ 27695,Tatyana_Smirnova@ncsu.edu \\ 2 Chemistry Division, Argonne National Laboratory, 9700 South Cass Avenue, Argonne, Illinois 60439
}

\begin{abstract}
Solvent effects on magnetic parameters of nitroxide spin labels in combination with side-directed spin labeling EPR methods provide very useful means for elucidating polarity profiles in lipid bilayers and mapping local electrostatic effects in complex biomolecular systems. One major contributor to these solvent effects is the hydrogen bonds that could be formed between the nitroxide moiety and water and/or the available hydroxyl groups. Here, formation of hydrogen bonds between a lipid bilayer spin probe -5-doxyl stearic acid, 5DSA - and hydrogen bond donors has been studied using high-frequency (HF) pulsed ENDOR and EPR. A hydrogen-bonded deuteron was directly detected in HF ENDOR $(130 \mathrm{GHz})$ spectra of 5DSA dissolved in several deuterated alcohols while the characteristic signal was absent in non-polar toluene- $d 8$. The length of the hydrogen bond, $1.74 \pm 0.06$ $\AA$, and its geometry were found to be essentially the same for all four alcohols studied, indicating that nearly identical hydrogen bonds have been formed regardless of the solvent dielectric constant. This strengthens a hypothesis that HF EPR spectra are exclusively sensitive to formation of hydrogen bonds and could be used for probing the hydrogen bond network in complex biomolecular assemblies and lipid bilayers with site-directed spin-labeling methods.
\end{abstract}

Electrostatic interactions and formation of hydrogen bonds are known to play the governing role in protein folding and function as well as in efficient charge separation and stabilization in enzymes, where hydrogen-bonding networks provide pathways for proton-coupled electron transfer processes. The same interactions also define insertion of membrane proteins into lipid bilayers. ${ }^{1}$ Detailed analysis of thermodynamics of insertion of small model peptides demonstrated that both favorable hydrophobic interactions and formation of hydrogen bonds between amino acid residues are required to lower the thermodynamic penalty of dehydrating the peptide bonds when placed inside the bilayer hydrocarbon core. ${ }^{2}$ Other sources of hydrogen bond donors are water molecules that have significant penetration into the lipid bilayer hydrocarbon core. ${ }^{3-5}$

Initial evidence of water penetration into lipid bilayers has been obtained by spin-labeling EPR. 3 Consequently, the bilayer hydration profile was derived from Gaussian fits of the x-ray diffraction data. 4,5 Further details on the bilayer water penetration were provided by electron spin echo envelope modulation spectroscopy (ESEEM) that detected two distinct populations of ${ }^{2} \mathrm{H} .{ }^{6}$ Based on the DFT calculations these components were assigned to water $\left({ }^{2} \mathrm{H}_{2} \mathrm{O}\right)$ molecules that are (i) hydrogen-bonded to spin-labeled lipids and (ii) free (unbound) water. The ESEEM water penetration profile was found to be similar to the polarity profiles derived 
earlier from measuring magnetic parameters, principal axis components $A_{z z}$ and $g_{x x}$, of spinlabeled stearic acids and lipids from rigid-limit continuous wave EPR spectra. $3,7,8$

Magnetic parameters of nitroxide spin labels are known to be sensitive to intermolecular interactions and, in particular, to hydrogen bonding and local solvent polarity. ${ }^{3}$ The solvent effects on nitroxide magnetic parameters can be easily detected by EPR at high magnetic fields from accurate measurements of both $A_{z z}$ and $g_{x x}$ providing better means to elucidate hydrogen bonding and electrostatic effects. ${ }^{7,8}$ Experimental and theoretical calibrations of electric field effects on nitroxide EPR spectra also were reported, ${ }^{9-11}$ indicating that formation of the hydrogen bond between nitroxide moiety and water should have the major effect on $g_{x x}$ while the dielectric constant provides a minor contribution affecting $A_{z z}$ and $g_{x x}$ in a correlated way. 11

It should be noted that while formation of hydrogen bonds between nitroxide moiety and water or alcohols has been implicated and discussed in many spin-labeling polarity studies, $3,6-8$, 11 none of these experiments provided detailed determination of parameters of the bond formed. We are interested in understanding the fundamental nature of the hydrogen bonds between the nitroxides and the likely donors and exploring these data for studying proteins in a lipid bilayer environment.

Here we report on high frequency (HF) ENDOR studies of a lipid bilayer spin probe -5-doxyl stearic acid (5DSA). This probe is routinely used in biophysical studies of phospholipid bilayers $^{3}$, is soluble in a variety of protic and aprotic solvents, and is structurally similar to spin-labeled lipids. A series of polar hydrogen-bond donor alcohols (such as deuterated 2propanol- $d 1$, butanol- $d 1$, ethanol- $d 1$, and methanol- $d 1$ ) and non-polar deuterated toluene- $d 8$ were used as solvents to determine $(i)$ the magnitude of magnetic interactions between the 5DSA electronic spin and deuterium of the hydroxyl group, (ii) existence of any correlations between the hydrogen bond length and the solvent polarity, and (iii) the hydrogen bond geometry.

HF D-band (130 GHz) EPR and ENDOR measurements were carried out with a spectrometer described previously. ${ }^{12}$ Field-swept echo-detected EPR spectra of 5DSA frozen solutions were recorded at $\mathrm{T}=25 \mathrm{~K}$ using a sequence of two pulses of 50 and $100 \mathrm{~ns}$ in length separated by a $350 \mathrm{~ns}$ delay and repeated at a $500 \mathrm{~Hz}$ rate. Typical spectra are shown in Fig. 1. Notably, while a single $g_{x x}$ component was observed for toluene (Fig. 1C) and THF (not shown) solutions, a partial splitting was detected for all alcohols studied (e.g., Fig. 1B). The low-field component characterized by $g^{0}{ }_{x x}$ was assigned to nitroxides that are not engaged in hydrogen bonds with solvent molecules and the high-field component $g_{x x}^{1}$ was attributed to nitroxide forming a single hydrogen bond. While some correlation between $g_{x x}$ components and $A_{z z}$ has been observed (not shown), the variations in $g^{0}{ }_{x x}$ and $g^{1}{ }_{x x}$ with the type of alcohol did not exceed the experimental error. Previously, a partial splitting in the $g_{x x}$ component of spinlabeled lipid bilayers was also observed; it was explained by the presence of free- and hydrogenbonded nitroxides in the bilayer. ${ }^{8}$

In order to directly probe the structure of 5DSA hydrogen bonds to the likely donors pulsed Mims-type ${ }^{2} \mathrm{H}$-ENDOR spectra were recorded from this protonated spin label at three magnetic positions corresponding to principal axis orientations of the $g$-matrix, $g_{x x}, g_{y y}$, and $g_{z z}$ (approximate positions are marked by dashed lines in Fig. 1). Selected experimental spectra are shown in Fig. 2. All experimental ${ }^{2} \mathrm{H}$-ENDOR spectra recorded in four alcohol solvents were found to be similar: a central peak observed at the ${ }^{2} \mathrm{H}$-Larmor frequency as superimposed with a doublet of symmetric lines (Fig. 2B). The relative intensity of the central peak with respect to the doublet varied with the solvent indicating that the former originated from the "matrix" deuterons. Intensity of the central line was found to correlate with the number of 
deuterons in the remote coordination spheres of the nitroxide moiety. Furthermore, for nonpolar solvent toluene- $d 8$ only the central matrix ENDOR line was detected, thus, reassuring the initial assignment. Therefore it was concluded that the doublet ENDOR line should arise from the splitting on the ${ }^{2} \mathrm{H}$ of the hydrogen bond. This splitting was found to be different for the $x$-, $y$-, and z-principal axis orientations (Fig. 2B). For all four alcohols the magnitudes of the splittings as well as line shapes were nearly identical. This indicates that both the strength of the hydrogen bond (i.e., spin to ${ }^{2} \mathrm{H}$ distance) and the bond geometry remain essentially the same for all four alcohol solvents studied. Simulations of the ENDOR spectra were carried out with a package "SimBud" provided by Dr. Astashkin (Univ. Arizona). ${ }^{13}$ In order to decrease the number of adjustable parameters several assumptions were made. Firstly, it was assumed that the deuteron of the hydrogen bond lies in the plane of the nitroxide ring. Secondly, the ${ }^{2} \mathrm{H}$ hyperfine and the nuclear quadrupole axes are collinear and determined by the direction of the hydrogen bond (Fig. 2A). Thirdly, the main contribution to the anisotropic hyperfine interaction arises from the unpaired electron spin density of the $p_{Z}$ orbitals of oxygen and nitrogen, set to $47.5 \%$ for both. Finally, direct calculations of the hyperfine tensor ${ }^{14}$ were carried out instead of an oversimplifying point dipole approximation. Under these assumptions, we have carried out simultaneous least-squares fitting of the ENDOR spectra for all three principal axis orientations of the 5DSA $g$-matrix.

The best-fit results summarized in Table 1 lead to the following conclusions: (i) the hydrogenbonded deuteron makes $\sim 120^{\circ}$ with the N-O bond, (ii) the bond length is $\sim 1.74 \AA$, and (iii) the best fit is obtained for a distribution of hyperfine coupling parameters, $\delta A_{i i}=0.1 \mathrm{MHz}$. While these distances and geometries agree well with theoretical studies ${ }^{11}$ and ENDOR data on hydrogen bonds formed by organic radical cofactors, 15,16 molecular modeling of the nitroxide-ethanol complex indicates a small potential van der Waals contacts of the alcohol with the nitroxide methyl groups.

Overall, we have provided direct experimental demonstration of hydrogen bonds formed by a lipid bilayer spin probe in hydrogen-bond-donor solvents and have determined the bond geometry. A correlation between the appearance of the high field $g_{x x}^{1}$ component (Fig. 1B) in the rigid limit HF EPR spectra and the characteristic doublet component in the ENDOR spectra has been observed. The length of the hydrogen bond and its geometry were found to be essentially the same for all alcohols studied, indicating that nearly identical hydrogen bonds have been formed regardless of the solvent dielectric constant. This strengthens a hypothesis that HF EPR spectra are exclusively sensitive to formation of hydrogen bonds and could be used to probe the hydrogen bond network in complex biomolecular assemblies and lipid bilayers by site-directed spin-labeling.

\title{
Supplementary Material
}

Refer to Web version on PubMed Central for supplementary material.

\begin{abstract}
Acknowledgements
The work at NCSU was supported by the NSF Grant MCB-0451510 to T.I.S. Work at ANL was supported by the U.S. Department of Energy, Office of Basic Energy Sciences, Division of Chemical Sciences, Geosciences, and Biosciences, under contract DE-AC02-06CH11357. A.I.S. and O.G.P. are thankful to NIH 1R01GM072897 for support.
\end{abstract}

\section{References}

1. Ladokhin AS, White SH. J Mol Biol 2001;309:543-552. [PubMed: 11397078]

2. White SH. Advances Prot Chem 2006;72:157-172.

3. Griffith OH, Dehlinger PJ, Van SP. J Memb Biol 1974;15:159-192. 
4. Wiener MC, White SH. Biophys J 1992;61:437-447.

5. Hristova K, White SH. Biophys J 1998;74:2419-2433. [PubMed: 9591668]

6. Erilov DA, Bartucci R, Guzzi R, Shubin AA, Maryasov AG, Marsh D, Dzuba SA, Sportelli L. J Phys Chem B 2005;109:12003-12013. [PubMed: 16852481]

7. Earle KA, Moscicki JK, Ge M, Budil DE, Freed JH. Biophys J 1994;66:1213-1221. [PubMed: 7518705]

8. Kurad D, Jeschke G, Marsh D. Biophys J 2003;85:1025-1033. [PubMed: 12885649]

9. Gullá AF, Budil DE. J Phys Chem B 2001;105:8056-8063.

10. Ding Z, Gullá AF, Budil DE. J Chem Phys 2001;115:10685-10693.

11. Owenius R, Engström M, Lindgren M, Huber M. J Phys Chem A 2001;105:10967-10977.

12. Poluektov OG, Utschig LM, Dubinskij AA, Thurnauer MC. J Am Chem Soc 2005;127:4049-4059. [PubMed: 15771542]

13. http://quiz2.chem.arizona.edu/epr/epr_000006.htm.

14. McConnell M, Strathdee J. Mol Phys 1959;2:129-138.

15. Sinnecker S, Reijerse E, Neese F, Lubitz W. J Am Chem Soc 2004;126:3280-3290. [PubMed: 15012159]

16. Wilson JC, Wu G, Tsai A, Gerfen GJJ. Am Chem Soc 2005;127:1618-1619. 

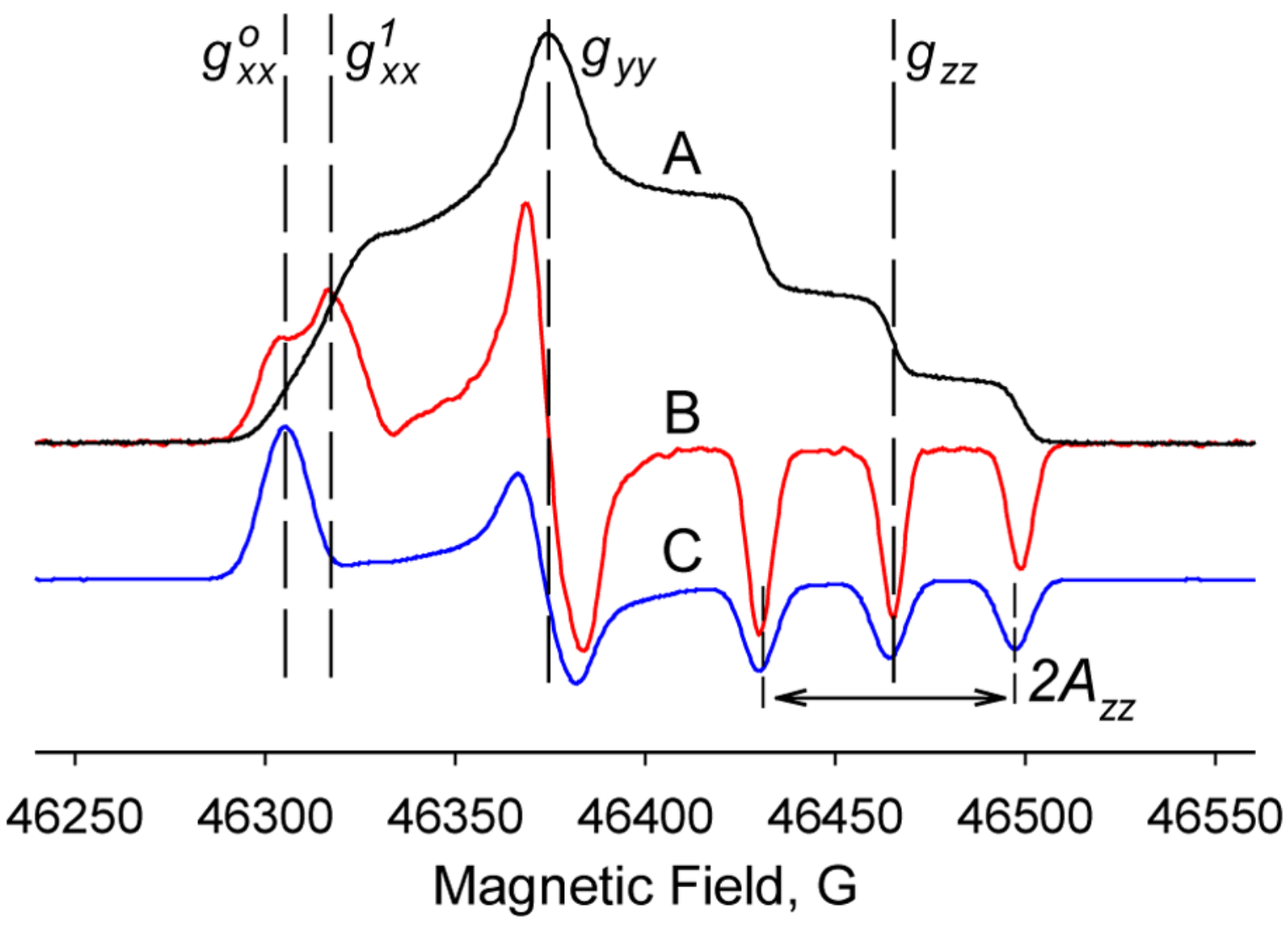

Figure 1.

Echo-detected $130 \mathrm{GHz}$ EPR spectra from 5DSA in deuterated 2-propanol- $d 1$ (A) and its first derivative (B), and in deuterated toluene- $d 8$ (C, derivative), $\mathrm{T}=25 \mathrm{~K}$. Dashed lines mark positions of principal axis components. Note partial splitting of $g_{x x}$ for 2-propanol- $d 1$ (B). 



Figure 2.

(A): Orientation of the 5DSA $g$-matrix and the bridging ${ }^{2} \mathrm{H}$ hyperfine $(A)$ and nuclear quadrupole $(\chi)$ tensors in the molecular frame of 5DSA coordinated with an alcohol molecule D-O-R'. Note that vectors $\boldsymbol{g}_{x x}, \boldsymbol{g}_{y y}, \boldsymbol{A}_{y y}, \boldsymbol{A}_{z z}$, lie in the same plane and that tensors $\boldsymbol{A}$ and are collinear. (B): Superimposed Mims-type HF ENDOR spectra of 5DSA in alcohols (black - 2propanol- $d l$; green - butanol- $d l$; cyan - methanol- $d l$; blue - etanol- $d l$ ), and toluene- $d 8$ (bold line for $g_{y y}$ orientation). Dashed red lines: least-squares simulations. 


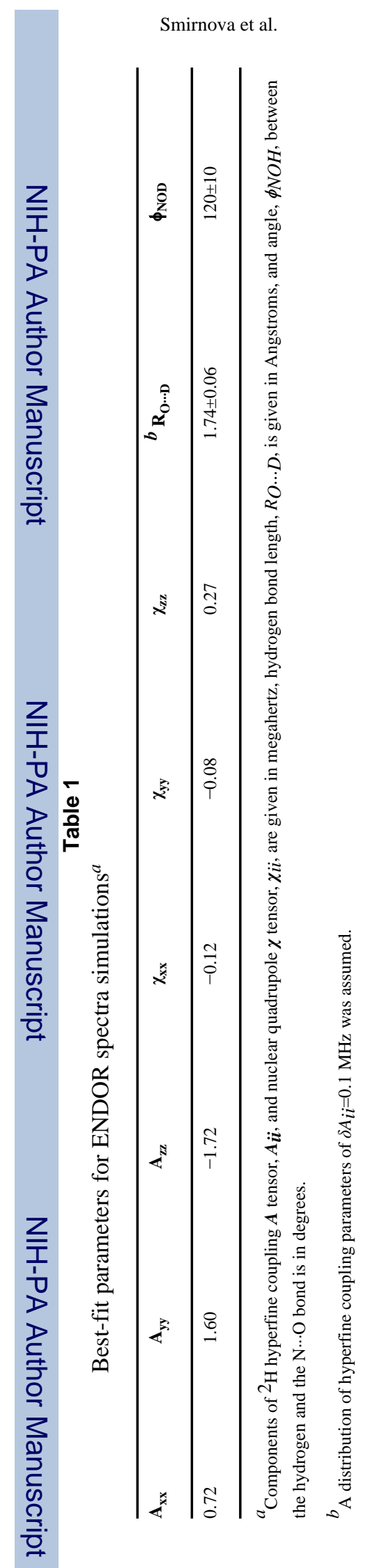

J Am Chem Soc. Author manuscript; available in PMC 2008 August 19. 